\title{
Role of Social Media in Healthcare Domain: An Integrated Review
}

\author{
Sridevi M. ${ }^{1}$, ArunKumar B.R. ${ }^{2}$ \\ ${ }^{1}$ Assistant Professor, Department of MCA, BMS Institute of Technology, Bangalore, India \\ ${ }^{2}$ Professor \& Head, Department of MCA, BMS Institute of Technology, Bangalore, India
}

\begin{abstract}
:
The ever increasing role of social media now-a-days in people's life created newer avenues for research in almost every domain. In the field of healthcare, social media and user-generated content are opening more opportunities for the researchers to kick-start their research by digging out patterns and trends from the millions of data points available and unveiling interesting correlations between the parameters that would affect the outcome of their research which would otherwise go unnoticed. Yet, there are some issues like ethical concerns, anonymity of data providers, and the apt approach to use the user-generated content, which have to be addressed through further research. The Hashtag project by organizations like Symplur Signals provides a free and open platform for the stakeholders in the healthcare domain that connects them to related communities and their conversations thus providing rich datasets of user-generated content for better research in healthcare social media.
\end{abstract}

Key Words: Social media, Healthcare, Ethical issues, patient community, Hashtag project.

\section{INTRODUCTION}

Social media is permeating into every domain like never seen before. In businesses, to improve the customer experience, social media is used to draw customer feedbacks, analyze them and identify useful patterns to understand the brand percolation into the market. Conventional feedback and review methods take long time to arrive at decisions to alter or continue the marketing strategy. This scenario can be changed drastically by use of social media to monitor the user generated content like peer reviews on products or services that open new doors to understand any requirements that tend to be unique as well as any drawbacks in the existing services that they provide. Social media is being a tool for individuals to share their views and lives, and plays a crucial role in initiating serious deliberations on business and technology. Shifting to the context of healthcare, the organizations have to understand how the chats on social media can be meaningfully harnessed to grow the business, visibility and open up new opportunities to research in social media monitoring related to healthcare. In order to avoid stagnation and becoming obsolete, the healthcare organizations have to actively take part in developing a social media policy which is of immense necessity today.

\section{TRENDS IN SOCIAL MEDIA SHAPING ITS POTENTIAL IN HEALTHCARE DOMAIN}

Social media serves as a vivid platform for various communities online to discuss about the trending topics. It can become a powerful tool for the patient empowerment by fostering their autonomy and by supporting them psychologically [1]. Social media also acts as an aid to the healthcare professionals by providing an opportunity to strengthen and stabilize the organization's position in the market [2]. Social media allows patients to get support and to add to offline information provided by healthcare professionals. Yet, it is always important to create online communication that do not exacerbate the health problems because social media is more prone to incorrect information being spread as anybody with access to the social media can "advice" on how to deal with adverse health conditions [3]. The effect of social media usage by patients for various health related reasons which can culminate into both benefits and challenges, have to be analyzed in depth for a better understanding of social media's potential in healthcare research. In [4], the authors provided an overview of the literature review on the articles published in and after 2010. Their survey mainly focused on the effects of usage of social media on patients and their relationship with healthcare professionals. They considered around 1,743 potentially related articles and narrowed their review to 22 articles after screening and filtering 
the articles based on preset selection criteria. The findings from the articles that were reviewed can be depicted with the help of the Figure 1. The patients use social media with an intention to meet an unfulfilled need. The types of usage of social media by patients can be categorized as social support that consists of emotional, informational, esteem and network support [5], and other types of usage namely social comparison and emotional expression.

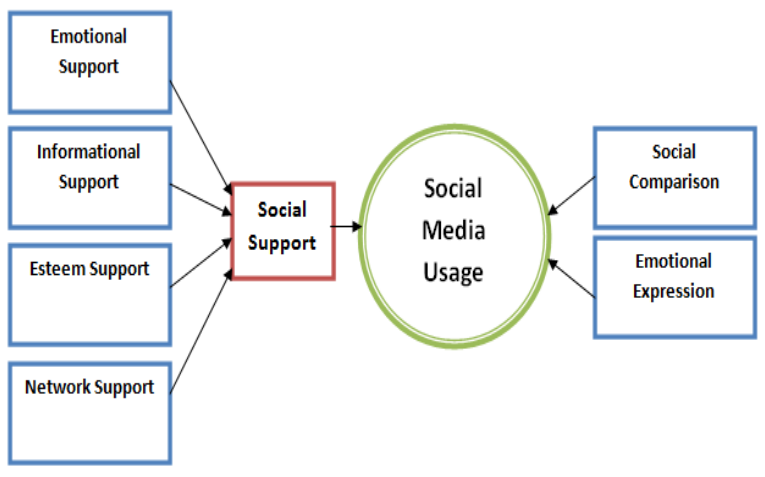

Figure 1. Types of Social Media usage by patients

Social media usage by patients provides them social support which is defined as the "process of interaction among relationship which is intended to improve sense of belonging, coping, esteem, and competence through actual or perceived exchanges of psychosocial resources [6]." Emotional support helps the patients to meet their effective or emotional needs with the discussion made online whereas esteem support aims to encourage an individual to take the appropriate action that is needed to live with their condition, successfully. This esteem support also helps patients to share experiences about a new treatment in order to find encouragement before starting the treatment [7]. The information support is helpful to patients who are newly diagnosed and are in a need for lot of inputs about their condition and available treatment options. This type of support can be provided by patients who were already dealing with the condition from a long time [8]. The network support answers patients that they are not alone and enables them to connect with a others in similar context [9] and developing relationships depending on the attributes shared between them [10].

Emotional expression and social comparison are the other benefits of social media usage by patients. Online expression of emotions provides the patients a unique opportunity to communicate without having to worry about the immediate reactions or feelings of those who coexist with them in their online community. Online communities allow the patients to open up without inhibitions that may be felt in sharing their experiences face to face [11]. Patients also use social media for comparing themselves with their counterparts in their community to understand about their present intensity of their condition or to know about how the treatments work. The graphical representation of the number of articles reviewed in [4] is as shown in Figure 2 which depicts the frequency of discussion of types of use of social media for health related purposes by the patients.
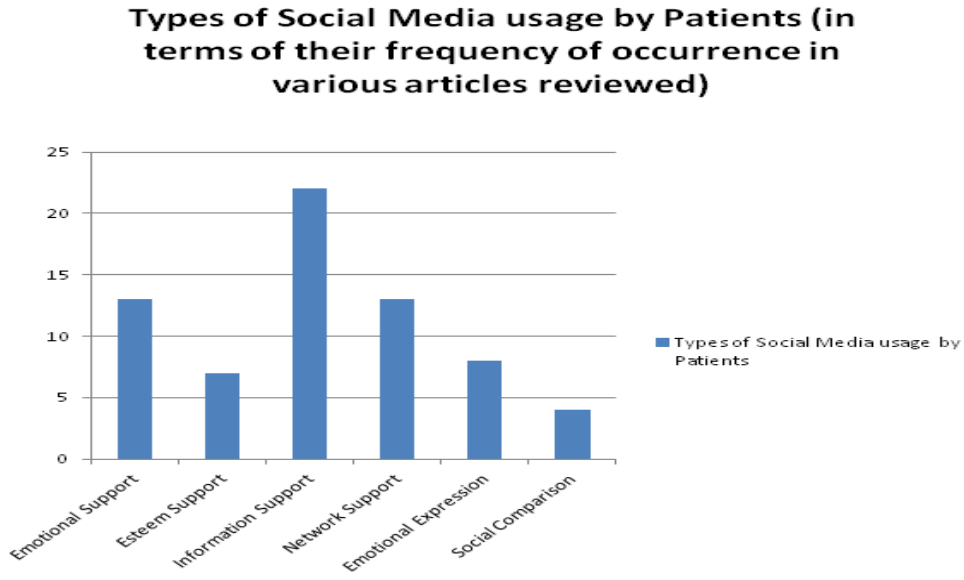

Figure 2. Graphical representation of the types of social media usage by patients for health related purposes (in terms of their frequency of discussion in various articles published between 2005 and 2016) 


\section{OUTCOMES OF VARIOUS TYPES OF SOCIAL MEDIA USAGE BY PATIENTS ON PATIENTS}

The outcomes or effects of various types of social media usage by patients on patients are analyzed in detail by the authors in [4]. Patient empowerment is the most common effect of social media usage which can be represented by three categories namely enhanced subjective well-being, improved control and self-management, and enhanced psychological well-being. The negative effects of the social media usage by patients were identified as diminished subjective well-being, privacy loss, social media addiction and promotional targeting. But, these negative effects are discussed in less number of articles indicating comparatively more positivity resulting from the usage of social media by patients related to healthcare. The topical frequency of the above said effects in the articles reviewed in [4] can be depicted with the help of the following bar graph in Figure 3. The graph clearly shows that the negative outcomes are considerably less when compared to the positive outcomes.

\section{Outcomes of social media usage by patients for health reated reasons (in terms of their discussion frequency in various articles reviewed)}

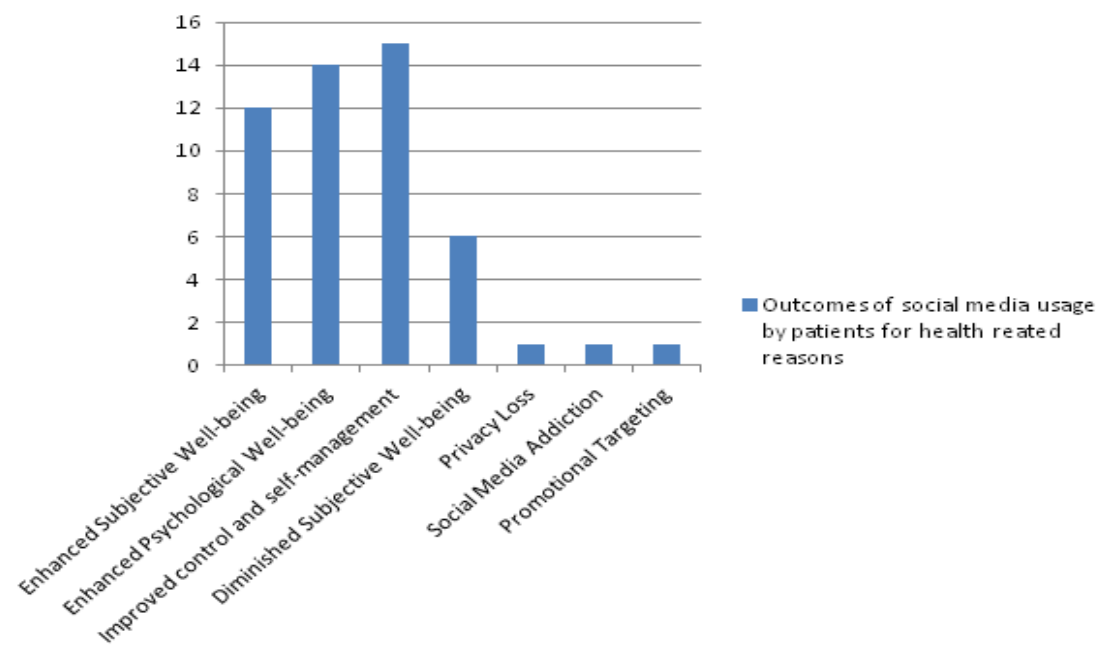

Figure 3. Outcome of social media usage by patients for health related reasons (in terms of their discussion frequency in various articles)

\section{SOCIAL NETWORK MODELLING AND ANALYSIS WITH BIG DATA SERVICE}

Online health platforms have provided avenues to patients to get useful information and share that information by exchanging their experiences among others with similar diseases. Such type of social networks will arm the researchers to conduct surveys and studies on the targeted groups of patients. At the same time, important factors like privacy preserving and reliability of data and user have to be considered to reap best out of the healthcare social networks.

In [12], the authors proposed a model for the social networks in the healthcare domain that combines the electronic health records (EHRs) and personal health records (PHRs) to support a network namely social health record (SHR) network which also enables preservation of privacy, data and user reliability in addition to the big data services provided to the participating organizations. They suggested the components for the multi-trust healthcare social network namely, individuals who can play the role of either patient or caregiver or both, healthcare providers and medical institutes, and social health records plus the virtual-network control centres (VCCs) that maintain the centralized database for pseudonymous accounts of the patients with trust control. This design is shown in the Figure 4. 


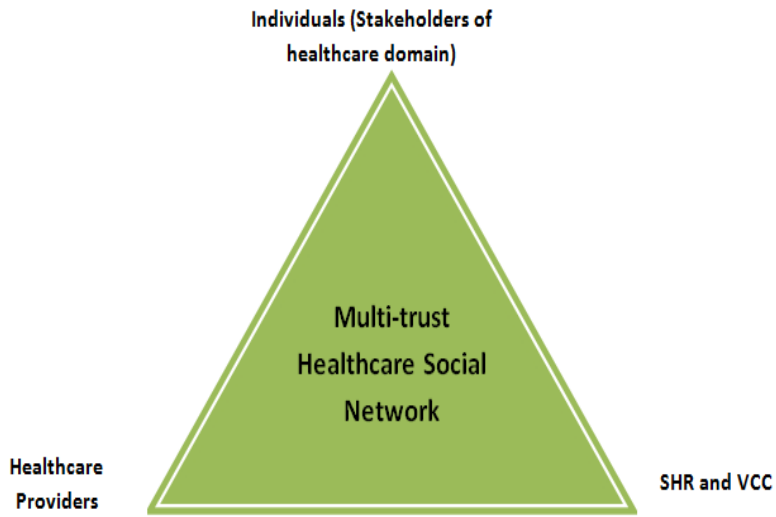

Figure 4. Multi-Trust Healthcare Social Network Components

The proposed model provides a platform to get second opinion for the patients which might be sought when they have to suffer from chronic diseases and conditions thereof for an enduring or long time. Also, it provides a platform for education of medical students who voluntarily participate in the healthcare social network. They can participate as special users to obtain extra knowledge and enrich their skills in treatment given to patients. The network can also serve as a platform for targeted surveys and other commercials. The authors in [12] also posed an open question as to how a system can detect or prevent falsification on the user-generated data that is self-reported in healthcare social network and thus paved a way for further research.

\section{ETHICAL ISSUES AND USABILITY OF PEER TO PEER EXCHANGED HEALTH INFORMATION FOR RESEARCH}

In contrast with the health research in online which the researcher decides the way of asking questions and participants choose how to answer them, using material or content that the people have posted already, for research opens up a good source of research data which can't be obtained elsewhere. This makes the decision making by the researchers difficult with respect to the ethical framework to be adopted, and the ways in which they analyze and visualize that data. This context raises new research questions about the appropriate approach to use the user-generated content in patient communities [13]. This research gap was addressed with a study on 30 people who were contacted via a discussion board for patients with diabetes [14]. Participants gave varied responses like participants who were aware of their information being shared in public domain felt no problem with aggregated information being used for analytics without their consent. But, there were diverged opinions on using information with a chance to identify the original contributors. Yet, some of the participants were of opinion that the usage of data generated by them should be acknowledged while others thought that their identity should be preserved.

\section{ROLE OF HASHTAG PROJECTS IN HEALTHCARE SOCIAL MEDIA}

Healthcare hashtag project by Symplur Signals is a free and open platform for caregivers, patients, doctors, advocates, and other providers that connect them to related communities and their conversations. The objective of this project is to make the use of social networking site like Twitter more accessible for providers and the healthcare community, by reducing the learning curve of Twitter with a database of relevant hashtags to follow, Symplur Signals help new and existing users alike to find the conversations that are of interest and importance.

Organizations like Symplur Signals organizes the Twitter hashtags that are in use which are relevant to the healthcare domain to -

- Allow exploration by either healthcare subject matter or a hashtag.

- Show hashtags that are related to one another in the healthcare field.

- Demonstrate the frequency of use of relevant hashtags so as to facilitate the selection of hashtags.

- Determines which of the healthcare hashtags are most popular at any point of time.

- Provide a live feed of conversations related to each hashtag so $\mathrm{s}$ to easily explore what is currently being discussed on Twitter in our healthcare topic of choice. 
- Encourage the visitors to vote on which hashtags are most useful in hope of bringing multiple conversations together.

The organizations like Symplur Signals promote understanding in depth of healthcare from the perspective of doctors, patients and other stakeholders by providing real-time access to insights from nearly a billion healthcare social media data points. Symplur Signals provides deep insights, rich datasets, powerful filtering, and algorithms with NLP (Natural Language Processing) capabilities optimize for our context of healthcare. Years of data can be analyzed from billions of healthcare related tweets with a trusted academic research platform.

\section{CONCLUSION}

The paradigm shift in the usage of social media by the stakeholders of the healthcare domain is leading to new avenues for research. The peer to peer patient information being exchanged online through patient communities, blogs, hashtag conversations etc., provide volumes of datasets of user-generated content for being analyzed and visualized to draw valuable conclusions to proceed with healthcare research. But, there are some open questions like the ethical framework to be designed and the appropriate approach to use the highly unstructured user-generated content in patient communities. The reliability of the user posted information also stands to be another question in healthcare social media research.

\section{REFERENCES}

[1]. Ho YX, OÇonnor BH, Mulvaney SA, "Features of online health communities for adolescents with Type 1 diabetes," West J Nurs Res., 2014; 36(9): 1183-98.

[2]. Williams J., "A New Roadmap for Healthcare Business Success," Journal of Healthcare and Finance Management Association, 2011; 65(5):62-9.

[3]. Carter M., "Medicine and the Media: How Twitter may have heped Nigeria contain Ebola,"Br Medical Journal 2014; 349.

[4]. Edin Smailhodzic et al., "Socia Media use in Healthcare: A Systematic Review of effects on patients and on their relationship with Healthcare Professionals," BMC Health Services Research (2016) 16:442, DOI 10.1186/s12913-016-1691-0.

[5]. Schaefer C, Coyne JC, Lazarus RS, "The Health-related Functions of Social Support," Journal of Behavioural Medicine, 1981; 4(4):381-406.

[6]. Cohen S, Underwood LG, Gottlieb BH, "Social Support Measurement and
Intervention: A Guide for Health and Social Scientists," New York, Oxford; 2000.

[7]. Coulson NS, "How do Online Patient Communities affect the experiences of Inflammatory Bowel Disease? An Online Survey," JRSM Short Reports, 2013; 4:1-8.

[8]. Mattson M, Hall JG, "Health as Communication Nexus: A Service-Learning Approach," Dubuque: Kendall Hunt; 2011.

[9]. Colineau N, Paris C, "Talking about your health to strangers: Understanding the use of Online Social Networks by Patients," New Rev Hypermed Multimed, 2010; 16(1): 14160.

[10]. Frost JH, Massagli MP, "Social uses of Personal Health Information within PatientsLikeMe, an Online Patient Community: What can happen when patients have access to one another's data," Journal of Medical Internet Research, 2008; 10(3):15.

[11]. Broom A., "The eMale: Prostrate Cancer, Masculinity, and Online Support as a challenge to Medical Expertise," Journal of Sociology, 2005; 41(1): 87-104.

[12]. Chunming Gao, Noriyuke Iwane, "A Social Network Model with Privacy Preserving and Reliability Assurance and its Applications in Healthcare," International Journal of Energy, Information, and Communications, Vol 6, Issue 1 (2015), pp. 45-58.

[13]. Whitehead LC, "Methodological and Ethical Issues in Internet-Mediated Research in the field of Health: An Integrated Review of the Literature," Social Science Med, 2007 August; 65(4): 782-91.

[14]. Track 3 Diabetes Planner - http:// www.track3.com/ android_diabetes_logbook/

\section{ABOUT THE AUTHORS}

M. Sridevi is Assistant Professor in the Department of MCA at BMS Institute of Technology, Bangalore, India. She is pursuing Ph.D. degree from VTU, Belagavi. Her research interests include Social Media Analytics, Data Mining, and Network Analysis. She published/presented 11 research papers in national/ international peer reviewed journals/ conferences.

Dr. ARUNKUMAR B.R., MCA, M.Phil (CS), M.Tech (CS\&E),PGDIPR[NLSIU], Ph.D (CS) from dept. of Computer Science, Dravidian University [A.P. Govt.], Kuppam, Professor and Head of the department, Master of Computer Applications, BMS Institute of Technology, Bangalore, Karnataka, India, Member BOS, VTU 
MCA , 2013-2016 has published nearly 32 research papers in National/International Journals and 11 papers in National/International Conferences including IEEE international conferences, wishes to place on record his sincere gratitude to BMS Education Trust, BMS Institute of Technology, Bangalore and expresses thanks to all those who helped in bringing out this paper. To his credit 5 candidates have earned their M.Phil (CS) degree and 5 candidates are pursuing their $\mathrm{Ph} . \mathrm{D}$ programme in computer Science/Applications. Dr.Arunkumar B R has got nearly 19 years of teaching experience and worked as Chairman, BOS, KSOU for UG/PG programmes in the year 2009-10. Dr.Arunkumar B.R has published several papers in his area of research namely: Data Mining, Data Analytics and Visualization, Wireless ad hoc networks, Computer Networks and Security, Cloud Computing, IPR \& Cyber laws and Software Engineering. 